\title{
ON THE APPROXIMATE SOLUTION OF LINEAR DIFFERENTIAL EQUATIONS WITH BOUNDARY CONDITIONS*
}

BY W. H. Mc EWEN

In a recent paper $\dagger$ the writer has studied the convergence of trigonometric and polynomial approximating sums to the solution of the $m$ th order linear differential system

$$
\begin{gathered}
L(y) \equiv \frac{d^{m} y}{d x^{m}}+Q_{1}(x) \frac{d^{m-1} y}{d x^{m-1}}+\cdots+Q_{m}(x) y=R(x), \\
U_{i}(y) \equiv \sum_{j=1}^{m}\left\{\alpha_{i}^{(j-1)} y^{(j-1)}(a)+\beta_{i}^{(j-1)} y^{(j-1)}(b)\right\}=h_{i}, \\
\quad(i=1,2, \cdots, m),
\end{gathered}
$$

the approximating sums being defined by a least $r$ th power criterion of best approximation. Thus in the polynomial case the approximating sum $P_{n}(x)$ was defined to be a polynomial of the $n$th degree which satisfies the boundary conditions $U_{i}\left(P_{n}\right)=h_{i}$ and at the same time minimizes the integral $\int_{a}^{b}\left|L\left(P_{n}\right)-R\right|^{r} d x$ in comparison with all other polynomials of that type, $r$ being a preassigned constant $>0$.

The purpose of this paper is to discuss the convergence question when a different criterion is used to define $P_{n}(x)$, namely, $P_{n}(x)$ is the approximating polynomial of the $n$th degree for the solution of the system (1) if it minimizes the expression

$$
\int_{a}^{b}\left|L\left(P_{n}\right)-R\right|^{r} d x+\sum_{i=1}^{m} C_{i}\left|U_{i}\left(P_{n}\right)-h_{i}\right|^{r_{i}}
$$

in comparison with all other polynomials of like degree, the $r$ 's and $C$ 's being given constants $>0$. Kryloff $\ddagger$ and Picone $\S$ have

* Presented to the Society, August 31, 1932.

$\dagger$ W. H. McEwen, Problems of closest approximation connected with the solution of linear differential equations, Transactions of this Society, vol. 33 (1931), pp. 979-997.

$\ddagger$ N. Kryloff, Les Problèmes Fondamentaux de la Physique Mathématique et de la Science d'Ingénieur, Monographie dans le Domaine de la Mathématique Appliquée, 1932, pp. 234-240.

$\S \mathrm{M}$. Picone, Sul metodo delle minime potenze ponderate e sul metodo di Ritz, etc., Rendiconti di Palermo, vol. 52 (1928), pp. 237-244. 
considered problems of minima similar to this but only for differential systems of the second order and with the additional restrictions, in the case of the former, that the $r$ 's be all alike and not less than 2, and in the case of the latter that they be all alike and not less than 1 . The methods used here are essentially different from those of the authors cited.

The fact that for each value of $n$ a polynomial $P_{n}(x)$ as defined above does exist, and when $r$ and the $r_{i}$ 's are $>1$ is unique, can be proved without difficulty by means of an argument which has been used elsewhere* in establishing theorems on existence and uniqueness relating to other problems of minima. Without dwelling further upon this point we shall proceed at once to a discussion of the convergence question.

In regard to the differential system we shall assume that it has a unique solution $y(x)$, or in other words that the corresponding reduced system is incompatible. It is understood, as in the paper first cited, that $R(x)$ and $Q_{1}(x), \cdots, Q_{m}(x)$ are continuous. Then, by Theorem $\mathrm{E}$ of that paper, there exist polynomials $p_{n}(x)$, each of degree indicated by its subscript, satisfying the boundary conditions $U_{i}\left(p_{n}\right)=h_{i}$, and such that for $a \leqq x \leqq b$

$$
\left|y^{(k)}(x)-p_{n}{ }^{(k)}(x)\right| \leqq \epsilon_{n}, \quad(k=0,1, \cdots, m),
$$

with $\lim _{n \rightarrow \infty} \epsilon_{n}=0$. For the convergence proof when $r<1$, we shall assume that $y(x)$ has further properties of regularity, which will automatically be realized if the coefficients in the differential equation satisfy suitable conditions as to continuity and existence of derivatives, so that according to the remark following Theorem $\mathrm{F}$ in the earlier paper it is possible in (3) to make $\lim _{n \rightarrow \infty} n^{2 / r} \epsilon_{n}{ }^{s}=0$, where $s$ is a preassigned positive number Under these hypotheses we shall prove that the $m$ quantities $\left|y^{(k)}(x)-P_{n}^{(k)}(x)\right|,(k=0,1, \cdots,(m-1))$, (and when $r<1$ the corresponding difference of the $m$ th derivatives also) will converge uniformly to zero on the interval $(a, b)$ as $n$ becomes infinite.

Let $F(x)=y(x)-p_{n}(x)$, where $p_{n}(x)$ is the polynomial described in the paragraph above. Then $F(x)$ satisfies the $m$ homogeneous boundary equations $U_{i}(F)=0$. Let $L(F)=Y(x)$, and let $\pi_{n}(x)$ be the approximating polynomial of the $n$th degree.

* See, for example, W. H. McEwen, loc. cit., pp. 981-982. 
defined with respect to the differential system $L(F)=Y$, $U_{i}(F)=0$, of which $F(x)$ is the unique solution. If $\gamma$ denotes the expression which in this case corresponds to (2), then

$$
\gamma=\int_{a}^{b}\left|L\left(\pi_{n}\right)-Y\right|^{r} d x+\sum_{i=1}^{m} C_{i}\left|U_{i}\left(\pi_{n}\right)\right|^{r_{i}}
$$

is a minimum for polynomials of the $n$th degree. But 0 may be regarded as a particular polynomial of the $n$th degree and moreover $U_{i}(0)=0$. Hence it follows that

$$
\gamma \leqq \int_{a}^{b}|Y|^{r} d x=\int_{a}^{b}|L(F)|^{r} d x \leqq K \epsilon_{n}^{r},
$$

where $K$ is a constant expressible in terms of the upper bounds which can be placed on the coefficients $Q_{i}(x), \cdots, Q_{m}(x)$ occurring in the left-hand side of the differential equation.

Since each term of $(4)$ is $\geqq 0$, it follows that each is $\leqq \gamma$. Hence

$$
\begin{aligned}
\int_{a}^{b}\left|L\left(\pi_{n}\right)-Y\right|^{r} d x & =\int_{a}^{b}\left|L\left(\pi_{n}-F\right)\right|^{r} d x \leqq \gamma, \\
\left|U_{i}\left(\pi_{n}\right)\right| & =\left(\frac{\gamma}{C_{i}}\right)^{1 / r_{i}}, \quad(i=1,2, \cdots, m) .
\end{aligned}
$$

By virtue of (5) these inequalities can be written

$$
\begin{aligned}
& \int_{a}^{b}\left|L\left(F-\pi_{n}\right)\right|^{r} d x \leqq K \epsilon_{n}{ }^{r}, \\
& \left|U_{i}\left(\pi_{n}\right)\right|=\left(\frac{K}{C_{i}}\right)^{1 / r_{i}} \epsilon_{n}^{s}, \quad(i=1,2, \cdots, m),
\end{aligned}
$$

where $s$ stands for the least of the positive numbers $1, r / r_{1}$, $r / r_{2}, \cdots, r / r_{m}$, and $\epsilon_{n}$ is assumed to be $<1$.

From this point on we consider separately the two cases $r \geqq 1$ and $r<1$.

The case $r \geqq 1$. Let $u(x)=F(x)-\pi_{n}(x)$ and $Z(x)=L(u)$. Then $u(x)$ satisfies the $m$ boundary equations $U_{i}(u)=-U_{i}\left(\pi_{n}\right)$, and hence is the unique solution of the differential system

$$
L(u)=Z(x), \quad U_{i}(u)=-U_{i}\left(\pi_{n}\right), \quad(i=1,2, \cdots, m) .
$$


Let $G(x, \xi)$ be the Green's function of the corresponding reduced system, and let $G_{1}(x), G_{2}(x), \cdots, G_{m}(x)$ be the respective solutions of the $m$ systems

$$
L(y)=0, \quad U_{i}(y)\left\{\begin{array}{l}
=0, \text { when } i \neq j, \\
=1, \text { when } i=j,
\end{array} \quad(i, j=1,2, \cdots, m),\right.
$$

Then

$$
\begin{array}{r}
u^{(k)}(x)=\int_{a}^{b} \frac{\partial^{k}}{\partial x^{k}} G(x, \xi) Z(\xi) d \xi-\sum_{i=1}^{m} G_{i}{ }^{(k)}(x) U_{i}\left(\pi_{n}\right), \\
\quad(k=0,1, \cdots,(m-1)) .
\end{array}
$$

Hence, by virtue of Hölder's inequality* and the fact that the functions $\partial^{k} G(x, \xi) / \partial x^{k}, G_{i}{ }^{(k)}(x)$ are bounded on $(a, b)$, we may write

$$
\left|u^{(k)}(x)\right| \leqq \bar{G}\left\{(b-a)^{(r-1) / r}\left[\int_{a}^{b}|Z(\xi)|^{r} d \xi\right]^{1 / r}+\sum_{i=1}^{m}\left|U_{i}\left(\pi_{n}\right)\right|\right\},
$$

where $\bar{G}$ is an upper bound of these functions.

Making use of (6), and the assumption made earlier that $\epsilon_{n}<1$ so that $\epsilon_{n}^{s}$ is the greatest of the numbers $\epsilon_{n}, \epsilon_{n}^{r / r_{i}}$, we can write

where

$$
\left|u^{(k)}(x)\right| \leqq K^{\prime} \epsilon_{n}^{s}
$$

$$
K^{\prime}=\bar{G}\left\{(b-a)^{(r-1) / r} K^{1 / r}+\sum_{i=1}^{m}\left(K / C_{\imath}\right)^{1 / r_{i}}\right\} .
$$

But since $\pi_{n}(x)$ and $P_{n}(x)$ are the respective approximating polynomials for $F(x)$ and $y(x)$, two functions whose difference is the polynomial $p_{n}(x)$, it is clear that

$$
u(x)=F(x)-\pi_{n}(x)=y(x)-P_{n}(x),
$$

and consequently

$$
\left|y^{(k)}(x)-P_{n}^{(k)}(x)\right| \leqq K^{\prime} \epsilon_{n}^{s},
$$

* We use this result in the form which states that if $\phi(x) \geqq 0$ and $r$ is any real number $\geqq 1$ then

$$
\int_{a}^{b} \phi(x) d x \leqq(b-a)^{(r-1) / r}\left[\int_{a}^{b}\{\phi(x)\}^{r} d x\right]^{1 / r} .
$$

It is at this point that we use the hypothesis in the definition of $P_{n}(x)$ that $r \geqq 1$. 
for $k=0,1, \cdots,(m-1)$ and for all values of $x$ on $(a, b)$. Thus the convergence of the approximating process is assured, since $\lim _{n \rightarrow \infty} \epsilon_{n}=0$.

The case $r<1$. $^{*}$ For the treatment of this case we shall establish the following result.

Auxiliary TheOREM. If the polynomial $\pi_{n}(x)$ satisfies the $m$ boundary equations

$$
U_{i}\left(\pi_{n}\right)=g_{i}, \quad(i=1,2, \cdots, m)
$$

and if $\delta=\max \left|L\left(\pi_{n}\right)\right|$ on $a \leqq x \leqq b$, then

(a) $\left|\pi_{n}{ }^{(k)}(x)\right| \leqq C\left[\delta(b-a)+\sum_{i=1}^{m}\left|g_{i}\right|\right],(k=0,1, \cdots, m)$, and if $Q_{1}(x), \cdots, Q_{m}(x)$ have bounded first derivatives,

$$
\left|\frac{d}{d x} L\left(\pi_{n}\right)\right| \leqq D n^{2}\left[\delta(b-a)+\sum_{i=1}^{m}\left|g_{i}\right|\right]
$$

for all values of $x$ on $(a, b), C$ and $D$ being constants independent of $n$ and independent of the coefficients in $\pi_{n}(x)$.

To prove conclusion (a) we proceed as follows. Let $Z(x)$ $=L\left(\pi_{n}\right)$. Then $\pi_{n}(x)$ is the unique solution of

$$
L(y)=Z(x), U_{i}(y)=g_{i}, \quad(i=1,2, \cdots, m),
$$

and hence

$$
\begin{aligned}
& \pi_{n}{ }^{(k)}(x)=\int_{a}^{b} \frac{\partial^{k}}{\partial x^{k}} G(x, \xi) Z(\xi) d \xi+\sum_{i=1}^{m} G_{i}{ }^{(k)}(x) g_{i}, \\
&(k=0,1, \cdots,(m-1)),
\end{aligned}
$$

where the $G$ 's are the same functions as defined in the first case. Hence

* The method of proof used in the first case is obviously not applicable when $r<1$ as it depended on a direct use of Hölder's inequality. The proof which we shall give here, however, can be applied equally when $r \geqq 1$, although the hypotheses which it imposes on the coefficients of the differential equation in order to insure a specified rate of convergence are somewhat more severe then in the first instance. As a point in its favor, however, it will be noted that the second method proves the convergence of $m$ derivatives as compared with $(m-1)$ by the first method. 


$$
\begin{aligned}
\left|\pi_{n}{ }^{(k)}(x)\right| & \leqq \bar{G}\left\{\int_{a}^{b}|Z(\xi)| d \xi+\sum_{i=1}^{m}\left|g_{i}\right|\right\} \\
& \leqq \bar{G}\left\{\delta(b-a)+\sum_{i=1}^{m}\left|g_{i}\right|\right\} .
\end{aligned}
$$

This holds for $k=0,1, \cdots,(m-1)$, but since

$$
\pi_{n}^{(m)}(x)=L\left(\pi_{n}\right)-Q_{1} \pi_{n}^{(m-1)}-\cdots-Q_{m} \pi_{n},
$$

a similar inequality holds for $k=m$. Hence there must exist a constant $C$ as stated in the theorem such that

$$
\left|\pi_{n}{ }^{(k)}(x)\right| \leqq C\left[\delta(b-a)+\sum_{i=1}^{m}\left|g_{i}\right|\right]
$$

for $k=0,1, \cdots, m$ and for all values of $x$ on $(a, b)$.

To prove conclusion (b) we note that $\pi_{n}{ }^{(m)}$ is a polynomial of the $n$th degree (in which the powers of $x$ from $n-m+1$ on are absent), and therefore by Markoff's theorem on the derivative of a polynomial

Hence

$$
\left|\pi_{n}{ }^{(m+1)}(x)\right| \leqq n^{2} C\left[\delta(b-a)+\sum_{i=1}^{m}\left|g_{i}\right|\right] .
$$

$$
\begin{aligned}
\left|\frac{d}{d x} L\left(\pi_{n}\right)\right|= & \mid \pi_{n}^{(m+1)}+Q_{1} \pi_{n}^{(m)}+\cdots+Q_{m} \pi_{n}^{\prime} \\
& +Q_{1}^{\prime} \pi_{n}^{(m-1)}+\cdots+Q_{m}^{\prime} \pi_{n} \mid \\
\leqq & D n^{2}\left[\delta(b-a)+\sum_{i=1}^{m}\left|g_{i}\right|\right]
\end{aligned}
$$

for values of $x$ on $(a, b)$, where $D$ is a constant of the character specified in the theorem.

To proceed with the proof of the convergence theorem, let us suppose for the time being that

$$
\epsilon_{n}{ }^{s}<\delta /[4(1+m) M],
$$

where $M$ is an upper bound of the coefficients in $L(y)$. Let $x_{1}$ be a point of the interval $(a, b)$ at which $L\left(\pi_{n}\right)$ attains its maximum value $\delta$. Then, by virtue of the mean value theorem and conclusion (b) of the auxiliary theorem, 


$$
\left|L\left(\pi_{n}(x)\right)-L\left(\pi_{n}\left(x_{1}\right)\right)\right| \leqq\left|x-x_{1}\right| D n^{2}\left[\delta(b-a)+\sum_{i=1}^{m}\left|g_{i}\right|\right] .
$$

But $g_{i}=U_{i}\left(\pi_{n}\right)$ and hence by $(6),\left|g_{i}\right| \leqq\left(K / C_{i}\right)^{1 / r_{i}} \epsilon_{n}{ }^{s}$. Therefore, making use of (7), we can write

$$
\left|g_{i}\right| \leqq\left(K / C_{i}\right)^{1 / r_{i}} \frac{\delta}{4(1+m) M}
$$

and so $\left|L\left(\pi_{n}(x)\right)-L\left(\pi_{n}\left(x_{1}\right)\right)\right| \leqq\left|x-x_{1}\right| D^{\prime} n^{2} \delta$, where

$$
D^{\prime}=D\left\{(b-a)+[4(1+m) M]^{-1} \sum_{i=1}^{m}\left(K / C_{i}\right)^{1 / r_{i}}\right\}
$$

is a constant independent of $n$.

We restrict our attention now to a small interval about $x_{1}$ in which $\left|x-x_{1}\right| \leqq 1 /\left[2 D^{\prime} n^{2}\right]$. In this interval

$$
\left|L\left(\pi_{n}(x)\right)-L\left(\pi_{n}\left(x_{1}\right)\right)\right| \leqq \delta / 2,
$$

and since $\left|L\left(\pi_{n}\left(x_{1}\right)\right)\right|=\delta$, it follows that

$$
\left|L\left(\pi_{n}(x)\right)\right| \geqq \delta / 2 \text {. }
$$

But $|L(F)| \leqq(m+1) M \epsilon_{n}<(m+1) M \delta /[4(m+1) M]=\delta / 4$. Hence in this interval

$$
\left|L\left(\pi_{n}\right)-L(F)\right| \geqq \delta / 4
$$

As the interval from $x_{1}$ to $x_{1}+1 /\left(2 D^{\prime} n^{2}\right)$ or else that from $x_{1}-1 /\left(2 D^{\prime} n^{2}\right)$ to $x_{1}$ is contained in $(a, b)$ for $n$ sufficiently large, it is clear that

$$
\int_{a}^{b}\left|L\left(\pi_{n}-F\right)\right|^{r} d x \geqq \frac{1}{2 D^{\prime} n^{2}}\left(\frac{\delta}{4}\right)^{r} .
$$

Hence

$$
\delta \leqq 4\left(2 D^{\prime}\right)^{1 / r} n^{2 / r}\left\{\int_{a}^{b}\left|L\left(\pi_{n}-F\right)\right|^{r} d x\right\}^{1 / r}
$$

and by virtue of (6) and the fact that $\epsilon_{n}<\epsilon_{n}{ }^{s}\left(\epsilon_{n}\right.$ being $\left.<1\right)$

$$
\delta \leqq 4\left(2 K D^{\prime}\right)^{1 / r} n^{2 / r} \epsilon_{n}{ }^{8} .
$$


This result was proved on the basis of assumption (7), but if (7) does not hold then clearly

$$
\delta \leqq 4(1+m) M \epsilon_{n}{ }^{s}<4(1+m) M n^{2 / r} \epsilon_{n}{ }^{8} .
$$

Hence if $E$ is the larger of the two constants $4\left(2 K D^{\prime}\right)^{1 / r}$ and $4(1+m) M$, we can write in any case

$$
\delta \leqq E n^{2 / r} \epsilon_{n}{ }^{8} .
$$

We make use of (8) and conclusion (a) of the auxiliary theorem to obtain an upper bound of $\left|\pi_{n}{ }^{(k)}(x)\right|$. Thus for $k=0,1, \cdots, m$,

$$
\begin{aligned}
\left|\pi_{n}^{(k)}(x)\right| & \leqq C\left\{(b-a) \delta+\sum_{i=1}^{m}\left|g_{i}\right|\right\} \\
& \leqq C\left\{(b-a) E n^{2 / r} \epsilon_{n}{ }^{s}+\sum_{i=1}^{m}\left|U_{1}\left(\pi_{n}\right)\right|\right\} \\
& \leqq C\left\{(b-a) E n^{2 / r} \epsilon_{n}^{s}+\left[\sum_{i=1}^{m}\left(K / C_{i}\right)^{1 / r_{i}}\right] \epsilon_{n}{ }^{s}\right\} \\
& =C^{\prime} n^{2 / r} \epsilon_{n}{ }^{s},
\end{aligned}
$$

where $C^{\prime}$ is a constant independent of $n$, the nature of which can be seen from the foregoing expression.

From this last result it follows that

$$
\begin{aligned}
\left|F^{(k)}(x)-\pi_{n}^{(k)}(x)\right| & \leqq\left|F^{(k)}(x)\right|+\left|\pi_{n}^{(k)}(x)\right| \leqq \epsilon_{n}+C^{\prime} n^{2 / r} \epsilon \\
& \leqq C^{\prime \prime} n^{2 / r} \epsilon_{n}{ }^{s},
\end{aligned}
$$

where $C^{\prime \prime}=\left(1+C^{\prime}\right)$ is a constant independent of $n$. But, as we have already observed in the first case, $F(x)-\pi_{n}(x)$ is identical with $y(x)-P_{n}(x)$ and hence

$$
\left|y^{(k)}(x)-P_{n}^{(k)}(x)\right| \leqq C^{\prime \prime} n^{2 / r} \epsilon_{n}^{8}
$$

for $k=0,1, \cdots, m$, and for all values of $x$ on $(a, b)$. Thus the approximating process converges if $\lim _{n \rightarrow \infty} n^{2 / r} \epsilon_{n}{ }^{8}=0$.

Mount Allison University,

Sackville, New Brunswick 